\title{
Papaya Genome: A Model for Tropical Fruit Trees and Beyond
}

\author{
Paul H. Moore • Ray Ming
}

Published online: 9 December 2008

(C) Springer Science + Business Media, LLC 2008

Papaya, Carica papaya L., a major fruit crop of the tropics, is featured in a series of articles in this, the first "special issue" of Tropical Plant Biology. One of the goals of TPB is to publish occasional special issues highlighting breakthroughs on a particular plant species or unifying problem of significance in plant tropical biology. It is fitting and with pride that we devote this first special issue of TPB to one of our personally favorite tropical plants, papaya. The timing of this particular special issue is based on the April 2008 report that the papaya genome has been sequenced [2] and the release of a large set of publicly available data, which is subsequently responsible for interesting and informative research such as that being reported in this issue. This special issue highlights specific examples of how a new and interesting plant genome sequence can open new avenues to better understand angiosperm genome evolution.

Papaya belongs to the order Brassicales that comprises 17 families including Caracacae, which contains papaya, and Brassicacae, the mustard or cabbage family known for its large and diverse collection of species (more than 3700 according to the Key Royal Botanical Gardens) including the model plant Arabidopsis thaliana. The placement of papaya and Arabidopsis deep within the order Brassicales allows one to use the extensive data and concepts developed extensively in Arabidopsis to discover possible

P. H. Moore $(\square)$

USDA-ARS, Pacific Basin Agricultural Research Center,

Hilo, HI 96720, USA

e-mail: Paul.Moore@ARS.USDA.GOV

R. Ming

Department of Plant Biology,

University of Illinois at Urbana-Champaign,

Urbana, IL 61801, USA

e-mail: rming@life.uiuc.edu gene function in papaya. More importantly, having the genome sequences of the closely related species provides information from one to help resolve unanswerable questions in the other. For example, which of the completely annotated genes of Arabidopsis are orthologs to which papaya genes? Analysis of syntenic blocks between Arabidopsis and papaya show that for single papaya genes there might be four corresponding genes in Arabidopsis [1]. The lack of multiple whole genome duplication events (polyploidy) in papaya make it a simple system for analysis and can help explain the course of genome evolution of orthologous genes in Arabidopsis.

Papaya, the fifth flowering plant to be sequenced, is also the first transgenic eukaryotic organism sequenced. Having the full genome sequence of a transgenic plant allows for detailed characterization of insert size, number, site, and function for a better understanding of genetic transformation on whole genome structure and function [7]. Among the plants sequenced, papaya has the fewest functional genes, apparently as the result of undergoing only one ancient genome triplication shared by most eudicots during its evolution. Compared to the other four sequenced plant genomes, the papaya genome count of 24,746 genes is $20 \%$ fewer than that of Arabidopsis, 34\% fewer than rice, $46 \%$ fewer than poplar, and $19 \%$ fewer than grape. The streamlined genome of papaya is already proving to be an excellent resource for comparative and functional analysis of both genes and repetitive elements and may provide clues about the minimum set of genes that are needed to be a flowering plant.

The relative simplicity of the papaya genome makes it ideal for detailed analysis of subsets of protein coding genes that may be involved in specific physiological and biochemical processes, anatomical and morphological characteristics, or environmental sensing pathways. Each 
of these areas is addressed in this special issue. For example, plants depend on cytochrome P450 (CYO) enzymes for nearly every aspect of their biology so that up to $1 \%$ of the protein coding genes might consist of CYP genes. Analysis of the 1415 CYP genes of the five sequenced genomes of higher plants and one of the primitive moss indicates that the 142 CYP genes of papaya have retained many of the ancestral characteristics and can act as the baseline for evaluation of the much more complex CYP genes of grape [4]. Another first for the papaya sequence is that papaya is the first fleshy fruit with a climacteric ripening pattern to be sequenced. The general pattern of having genes homologous to those of Arabidopsis and tomato for fruit size, shape, ethylene synthesis and response, respiration, chlorophyll degradation, and carotinoid synthesis, but in smaller numbers than in Arabidopsis and tomato, again indicates the potential for using papaya to understand the complex regulatory networks active in processes such as fruit ripening [5]. The circadian transcriptional networks, previously described in Arabidopsis and other plant sequences, are strongly conserved in papaya despite its tropical habit and distinct life-style. This suggests that circadian timing has played a major role in the evolution of plant genomes, consistent with the selective pressure of anticipating daily environmental changes [9].

A little more than half $(52 \%)$ of the papaya genome consists of repetitive sequences, the majority of which are transposable elements [3]. Telomeres of papaya, like those of most other plant species consist of TTTAGGG repeats, but, in contrast to members of the closely related Brassicaceae family, the telomeres of papaya are approximately ten-fold longer and the genes involved in telomere maintenance and chromosome end protection exist as single copies, as opposed to the multiple copies in Arabidopsis [6]. The most abundant tandem repeats are microsatellites, but they represent only $0.19 \%$ of the papaya genome. A genome-wide analysis of microsatellites [8] revealed a density of one for every $0.7 \mathrm{~kb}$ of genome sequence. The abundance of microsatellites enabled the development of a high density linkage map that is sufficient for genome assembly and for mapping of most papaya traits.
Tropical fruits are rich in nutrition and many have medicinal properties. Although papaya is not a typical model species, it can serve as a genomic model for characterizing biological features relevant to tropical environment and for dissecting the genetic bases of nutritional and medicinal profiles of tropical fruits and other fruit and tree species in general. After you read the articles in this issue of TPB, we hope you agree that such a focus is valuable. We welcome suggestions for future topics to treat in this manner.

\section{References}

1. Lyons E, Pedersen B, Kane J, Freeling M (2008) The value of nonmodel genomes and an example using SynMap with CoGe to dissect the hexaploidy that predates the rosids. Trop Plant Biol (this issue)

2. Ming R et al (2008) The draft genome of the transgenic tropical fruit tree papaya (Carica papaya Linnaeus). Nature 452:991-996

3. Nagarajan N, Navajas-Perez R, Pop M, Alam M, Ming R, Paterson A, Salzberg S (2008) Genome-wide analysis of repetitive elements in papaya. Trop Plant Biol (this issue)

4. Nelson DR, Ming R, Alam M, Schuler MA (2008) Comparison of cytochrome P450 genes from six plant genomes. Trop Plant Biol (this issue)

5. Paull RE, Irikura B, Wu PF, Turano H, Chen NJ, Blas A, Fellman J, Gschwend AR, Wai CM, Yu Q, Presting G, Alam M, Ming R (2008) Fruit development, ripening and quality related genes in the papaya genome. Trop Plant Biol (this issue)

6. Shakirov EV, Salzberg SL, Alam M, Shippen DE (2008) The analysis of Carica papaya telomeres and telomere-associated proteins: insights into the evolution of telomere maintenance in Brassicales. Trop Plant Biol (this issue)

7. Suzuki JY, Tripathi S, Fermín G, Hou S, Saw J, Ackerman CM, Yu Q, Schatz MC, Pitz KY, Yépes M, Fitch MMM, Manshardt RM, Slightom JL, Ferreira SA, Salzberg SL, Alam M, Ming R, Moore PH, Gonsalves D (2008) Characterization of insertion sites in Rainbow papaya, the first commercialized transgenic fruit crop. Trop Plant Biol (this issue)

8. Wang J, Chen C, Na JK, Yu Q, Hou S, Paull RE, Moore PH, Alam M, Ming R (2008) Genome-wide comparative analyses of microsatellites in papaya. Trop Plant Biol (this issue)

9. Zdepski A, Wang W, Priest HD, Ali F, Alam M, Moclker TC, Michael TP (2008) Conserved daily transcriptional programs in Carica papaya. Trop Plant Biol (this issue) 